and biochemistry methods. Having two PH domains, MPRIP has an affinity to Phosphoinositols and as a key regulator of protein phosphatase 1 , it is a potential mediator for dephosphorylation of Pol II and splicing machinery (Mulder et al., 2003). Methods: We have first investigated the localization of the endogenous MPRIP by super-resolution microscopy (STED) and visualized that it forms nanoscale foci in the nucleoplasm, which partly coalesce with PIP2 containing structures in nucleus, particularly with nuclear speckles and sub-population of Nuclear Lipid Islets (NLIs) (Sobol et al., 2018). To study the dynamics of endogenous MPRIP nanoscale foci is extremely challenging, therefore we employ GFPMPRIP overexpression, in order to address question about the inner dynamics and mobility of MPRIP induced condensates. Results: The transient transfections experiments showed that highly expressing protein undergoes phase separation by accumulating in nucleus where it forms condensates that are up to $5 \mu \mathrm{m}$ in diameter. We presume that this formation is mediated by the $\mathrm{C}$ Terminal- Intrinsically Disordered Region (IDR). The Fluorescence recovery after photobleaching (FRAP) and live-cell imaging experiments uncovered their rapid motion, of which we have quantified the diffusion coefficients as a way to comprehend how the protein might act at endogenous levels. Conclusion: Here, we showed that MPRIP, an actin regulatory protein, is present in mammalian nucleus and partly localize to proximity of PIP2 rich structures. We hypothesize that MPRIP might regulate transcription by recruiting its known interactor, Protein phosphatase 1 , to the vicinity of NLIs. Altogether, these data indicates that MPRIP might be an impor- tant regulator of Pol II transcription, while the exact pathway remains to be enlightened.

This study was supported by the Czech Academy of Sciences (JSPS-18-18); Grant Agency of the Czech Republic (16-03346S, 17-09103S, 15-08738S).

References: Mulder, J., Poland, M., Gebbink, M.F.B.G., Calafat, J., Moolenaar, W.H., Kranenburg, O., 2003. p116Rip Is A Novel Filamentous Actin-binding Protein. J. Biol. Chem. 278, 27216-27223. Sobol, M., Hozak, P., 2018. Nuclear phosphatidylinositol 4,5-bisphosphate islets contribute to efficient RNA polymerase II-dependent transcription. Journal of Cell Science 131, jcs211094. https:// doi.org/10.1242/jcs.211094

doi: http://dx.doi.org/10.7124/bc.0009D5

\section{B-4. Abiotic stress effects - a model for studying the structure of plant nucleoloneme and chromatin transformations}

\author{
E. N. Baranova, L. I. Fedoreyeva, A. A. Gu- \\ levich \\ All-Russia Research Institute of Agricultural Bio- \\ technology, Timiriazevskaya 42, 127550 Moscow, \\ Russia \\ greenpro2007@rambler.ru
}

The study of ultrastructural transformations of the nuclear domains of cells of actively dividing tissues of the meristem under the influence of abiotic environmental factors such as cold, salinity, changes in the magnetic field parameters accompanying the change in the level of gene expression and chromatin modification allow visualizing these processes. We present the details of the process of structural organization, formation and disassembly of the nucleolus, chromatin condensation during the progression 
of the cell cycle studied by the method of classical TEM in the cells of the meristematic zone of the wheat root. The transformations of peripheral chromosomal material (PCM) in telophase, nucleolonema of nucleoli in interphase and prophase, chromatin transformation (condensation and decondensation) under conditions of the geomagnetic field, weakened magnetic field and static magnetic field at low positive temperatures and salinity were investigated and analyzed. Using an analysis of the dynamics of structural modifications of proliferating nuclei, there was a disturbance in the formation of the chromonema and condensed chromatin in the middle interphase, a change in the structural organization of the nucleolus during the transition from late interphase to prophase, as well as a change in the structure of PCM, pre-nucleolus and chromatin in telophase. A scheme has been proposed for the formation of a specific nucleolonemal and chromonemal structure and its influence on the structural organization of chromosomes in anaphase and telophase. The nature of this structure identified earlier in normal and stressful conditions in rye and barley $[1,2]$ and wheat in the acidic and alkaline reaction of the environment and cold $[3,4]$ is discussed. The studied effects associated with the transformation of the structural organization of the nuclei caused a change in the expression level of the genes of some DNA methyltransferases, probably related to the epigenetic regulation of the genome. The proposed mechanisms of action of low positive temperatures, salinization, magnetic field on the nuclear compartment and its domains, regulation of chromatin decondensation, export of pro-ribosomal particles under stress, and the fine structure of the nucleolonema are discussed.
This work was supported by the Russian Science Foundation (grant 18-016-00150) and by the state assignment AAAA-A18-118051890089-0.

Referenses: 1.Baranova EN, Baranova GB, Kharchenko PN (2011) Effect of weak magnetic field and low positive temperature on chromatin and nucleolus ultrastructure of rye and barley. Russ Agric Sci 37: 453-461. 2. Wang J, Zhang F (2015) Nucleolus disassembly and distribution of segregated nucleolar material in prophase of root-tip meristematic cells in Triticum aestivum L. Arch Biol Sci 67: 405-410. 3. Avetisova LV, Kadykov VA (1985) Ultrastructure of Wheat Apical Meristem Cells at Low Positive Temperatures, 1: Nuclear Structure. Tsitologiya, 27: 28-32. 4. Baranova EN, Gulevich AA (2009) Structural organization of nuclei and nucleoli of wheat shoot and root meristem during germination under alkaline $\mathrm{pH}$ conditions. Russ Agri Sci 35: 11-14.

doi: http://dx.doi.org/10.7124/bc.0009D7

\section{B-5. The Perichromatin Region: a crossroad of events}

\author{
M. Biggiogera, S. Siciliani, L. Zannino \\ Dipartimento di Biologia e Biotecnologie "L. Spall- \\ anzani", University of Pavia, Italy \\ marcobig@unipv.it
}

The Perichromatin Region (PR) is the 200-nmthick rim surrounding the condensed chromatin areas in the interphase cell nucleus. First described by Bernhard (1969), PR has been studied for years and several fundamental processes have been shown to take place there: DNA replication, repair, transcription, co-transcriptional splicing. Recently we have pointed out that possibly other mechanisms occur in that region (Masiello et al. 2018). We have studied the PR in several cell and tissue models, both in physiological conditions and after drug treatment. We can confirm that not only methylation on cytosine in DNA and RNA 